\title{
Erratum
}

\section{Equations for the analysis of the light curves of extra-solar planetary transits}

\begin{abstract}
A. Giménez
Research and Scientific Support Department, European Space Agency, ESTEC, Keplerlaan 1, 2200 AG Noordwijk ZH, The Netherlands

e-mail: agimenez@rssd.esa.int

A\&A 450, 1231-1237 (2006), DOI: 10.051/0004-6361:20054445

Key words. techniques: photometric - Stars: binaries: eclipsing - stars: planetary systems - errata, addenda

The argument $\theta$ in Eq. (9) of Giménez (2006a) does not denote the orbital phase as in the rest of the paper. Equation (9), like Eq. (14) of Giménez (2006b), describes the projected separation between the centre of the star and the planet in terms of the semi-major axis for eccentric orbits. In these equations, $\theta$ denotes an auxiliary angle so that the true anomaly can be reckoned from the centre of the transit. As mentioned in the paper, this angle is shown in Fig. 1 by Giménez \& Garcia-Pelayo (1983) and is in fact given by $v+\omega-\pi / 2$, where $v$ represents the true anomaly and $\omega$ the position of the periastron. In case of circular orbits there is of course no difference between $\theta$ and the orbital phase. Fortran subroutines for the computation of light curves and the Rossiter effect, simultaneously, are available at http://thor.ieec.uab.es/LRVCode/
\end{abstract}

\section{References}

Giménez, A. 2006a, A\&A, 450, 1231

Giménez, A. 2006b, ApJ, 650, 408

Giménez, A., \& Garcia-Pelayo, J. M. 1983, Ap\&SS, 92, 203

Article published by EDP Sciences and available at http://www.aanda.org or http://dx.doi.org/10.1051/0004-6361:20054445e 